\title{
Minimum-Delay Overlay Multicast
}

\author{
Kianoosh Mokhtarian and Hans-Arno Jacobsen \\ Department of Electrical and Computer Engineering \\ University of Toronto \\ Toronto, ON, Canada
}

\begin{abstract}
Delivering delay-sensitive data to a group of receivers with minimum latency is a fundamental problem for various applications. In this paper, we study multicast routing with minimum end-to-end delay to the receivers. The delay to each receiver in a multicast tree consist of the time that the data spends in overlay links as well as the latency incurred at each overlay node, which has to send out a piece of data several times over a finite-capacity network connection. The latter portion of the delay, which is proportional to the degree of nodes in the tree, can be a significant portion of the total delay as we show in the paper. Yet, it is often ignored or only partially addressed by previous multicast algorithms. We formulate the actual delay to the receivers in a multicast tree and consider minimizing the average and the maximum delay in the tree. We show the NP-hardness of these problems and prove that they cannot be approximated in polynomial time to within any reasonable approximation ratio. We then propose a number of efficient algorithms that heuristically build a multicast tree in which the average or the maximum delay is minimized. These algorithms together cover a wide range of overlay sizes and both versions of our problem. The effectiveness of our algorithms is demonstrated through comprehensive experiments on different real-world datasets and using various overlay network models. The results confirm that our algorithms can achieve much lower delays (up to 60\% less) and up to orders of magnitude faster running times (hence supporting larger scales) than previous minimum-delay multicast approaches.
\end{abstract}

\section{INTRODUCTION}

Minimum-delay routing of data in overlay networks is a fundamental problem for several distributed applications. For instance, consider a delay-sensitive event notification system in which an event generated at a node needs to be signalled to a large group of monitoring nodes with minimum latency, e.g., a Distributed Interactive Simulation (DIS) software for military systems [1], [2], financial trading through large groups of globally-interlinked computer systems [3], [4], or massive multiplayer online games [5], 
[6]. Also note that the group of receivers corresponding to a source node in these systems may not be constant over time, such as a dynamic agent in a virtual environment (e.g., online game) moving across the Area of Interest (AOI) of other entities [7], [8].

Therefore, forming overlay multicast groups (which nodes should join and leave) and maintaining the corresponding state information in the intermediate overlay nodes, as in several classic multicast techniques [9]-[12], is not an efficient solution. A naive alternative approach is to send data directly from the source to each receiver. This solution, however, is not scalable since it requires each node to have (and constantly monitor the state of) a connection to every other node in the network. Moreover, this approach can incur long delays, because a node has a finite-bandwidth connection to the network, over which several copies of the same data should be sent. To avoid these problems, nodes can be connected through a mesh overlay. Then, source-based minimum-delay multicast trees can be calculated on demand for transmitting the data [13]-[16], which is the problem of our interest in this paper.

While the primary goal of typical shortest-path multicast routing schemes has been to minimize the total link-by-link delay experienced by packets, we observe that a significant portion of the total end-toend delay in a multicast scenario is the delay incurred at overlay nodes. This is because each intermediate node has to send out several copies of the same packet through a finite-capacity network connection. This node-incurred delay, which is in proportion to the degree of the node in the routing tree, is in addition to the delay occurring in overlay links and can even dominate it as we show shortly. In particular, this issue becomes more critical in large-scale overlay networks with strong connectivity. In these networks, as intuitively expected, most shortest paths consist of a few hops only [17], [18], hence leading to large node degrees in a multicast tree.

To get a numeric intuition on this problem, suppose we would like to deliver one packet of $1.2 \mathrm{~KB}$ (10 Kbits) to 1000 nodes in an event notification overlay. Assume that overlay nodes have a $10 \mathrm{Mbps}$ Internet connection and are on average handling 20 concurrent sessions; hence it takes $(0.001 \mathrm{~s}) \times 20=20 \mathrm{~ms}$ for a node to send out one copy of the packet. Also assume that the delay between every pair of nodes is $100 \mathrm{~ms}$, and the average shortest path length is 3 hops in the overlay (i.e., a delay of $300 \mathrm{~ms}$ ). Thus, the average degree of nodes in the multicast tree is about $1000^{1 / 3} \simeq 10$, and the average delay incurred by each node to forward the packet is average $e_{i=1, \ldots, 10}(i \times 20 \mathrm{~ms})=110 \mathrm{~ms}$, i.e., a total node-incurred delay of $330 \mathrm{~ms}$ in a typical 3-hop path. Note that the total link-by-link delay of such path was only 300 ms.

Yet, the problem with delays incurred by node degrees in an application-layer multicast tree is often ignored [16], [19]-[22] or only partially addressed by previous works, such as bounding node degrees 
in a tree to predefined thresholds [14], [23]-[26]. The problem with large node degrees, however, is of the same type as the shortest-path routing problem-minimizing the incurred delay. It thus needs to be considered together with link delays in the routing decisions, rather than as a separate problem and at the coarse grain of being or not being within a threshold.

In this paper, we study the overlay multicast routing problem for minimizing the actual end-to-end delay. In particular, the contributions of this paper are as follows. We first formulate the two problems of minimizing the average and the maximum delay in multicast trees, and we prove their NP-hardness as well as their inapproximability to within any reasonable ratio. That is, we show that no polynomial-time approximation algorithm can guarantee any better delay than several times beyond the optimal value. We then present a set of efficient algorithms for heuristically building a multicast tree with minimum average (or minimum maximum) delay. These algorithms together support a wide range of overlay sizes for both minimum-average and minimum-maximum applications. To demonstrate the effectiveness of these algorithms, we conduct an extensive evaluation study on different real-world datasets and using three different overlay network models. We show that the actual delay observed by multicast receivers can be reduced by up to $60 \%$, and the calculation time for multicast trees by up to orders of magnitude (hence supporting larger scales), if our algorithms are employed.

In the remainder of this paper, we first summarize the related work in Section II. We then formally define our multicast problems in Section III and review the routing model on which our algorithm is built. Section IV presents our algorithms, followed by a thorough evaluation study in Section V. We conclude the paper in Section VI.

\section{RELATED WORK}

The problem of our interest in this paper is to deliver data with minimum end-to-end delay from a given source to a set of receivers on a mesh overlay. Compared to arranging nodes in fixed overlay multicast trees [23], [25], calculating on-demand per-source trees on a well connected mesh overlay provides much higher flexibility in selecting paths and better resilience against dynamics of the network [11], [20]. Moreover, as discussed earlier we consider source-based multicasting, in which the intermediate nodes do not need to keep any per-session state information or to perform route calculations for each message. Instead, the source calculates the routing tree and embeds it in the message (e.g., using Bloom Filters [15]), so the intermediate nodes only perform simple forwarding.

Most overlay multicast algorithms only minimize the link-by-link distance to the receivers [16], [19][22]. However, as discussed earlier, a significant factor in the actual end-to-end delay is the delay incurred 
at overlay nodes that send out each message several times. There have been a number of previous works that did consider the node degree problem [14], [24], [26]-[28], but they only try to find a routing tree in which the degree of nodes is bounded to predefined thresholds. Ito et al. [28] analyzed several forms of the multicast tree problem, and showed that it is NP-Complete to find various forms of degreebounded trees, such as one with minimum total distance or one with minimum distance to the farthest receiver. Heuristic algorithms for constructing degree-bounded multicast trees [14], [27] and degreebounded minimum-diameter spanning trees [24], [29] have been proposed. However, it is not clear how these degree bounds are selected in practice; for instance, a fixed bound of 10 is used in [29]. In [26] it is proposed to set the degree bounds based on the level of the node in the tree. Nevertheless, these works only aim at bounding node degrees to given thresholds. Rather than bounding degrees at such coarse grain (i.e., only to a threshold), we capture the delay caused by node degrees together with the delay incurred at overlay links as a single delay cost and minimize it.

The only previous work considering this problem, to the best of our knowledge, is done by Brosh et al. [13] who propose an approximation and a heuristic algorithm for minimizing the maximum end-toend delay in a multicast tree (the problem with minimum-average delay is not considered). However, the proposed approximation algorithm and its bound only correspond to the special case of broadcasting a message in a complete overlay graph, whereas often in practice neither the overlay network is a complete graph nor all messages are destined to all nodes. Furthermore, even for this special case the approximation factor is $O(\log n)$ and $O(\log n / \log \log n)$ for directed and undirected overlay graphs, respectively, which is a considerable amount. In fact, the heuristic algorithm proposed by the authors (with no approximation guarantee) provides lower end-to-end delays than the approximation algorithm while being also more efficient in running time [13]. Nevertheless, the achieved delay and the running time of this algorithm are significantly larger than our algorithms.

We also note that an important factor determining the scalability of a multicast scheme is the underlying routing protocol. The common approach used in [13] and several other works is link-state based routing [12], [15]-[17], [19], [22], which allows all nodes to know the full topology of the network while suffering from high overhead and limited scalability (as we will show). Our multicast scheme, on the other hand, is based on a variant of distance-vector routing and can be up to orders of magnitude more scalable. 
TABLE I

NOTATIONS USED IN THIS PAPER.

\begin{tabular}{|l|l|}
\hline Notation & Description \\
\hline$G, V, E, N$ & Overlay graph $G=(V, E) . N=|V|$. \\
\hline$G_{P V}$ & Partial view of overlay graph based on path vectors. \\
\hline$T, s, R$ & The routing tree, the source node, and the set of receiver nodes, respectively. \\
\hline$z$ & Size of the message being distributed. \\
\hline$\Delta_{u}(z)$ & Time it takes for node $u$ to send out a message of size $z$. \\
\hline$w(u, v)$ & Delay of overlay link $(u, v)$. \\
\hline$c_{T}(u, v), \hat{c}_{T}(u, v)$ & Exact and expected value of the actual delay from $u$ to $v$ in tree $T$. \\
\hline$d_{T}, d_{G}(u)$ & Degree of node $u$ in tree $T$ or graph $G$. \\
\hline$q_{u, T}(v)$ & The turn of $v$ among the children of $u$ in $T$. \\
\hline$t_{T}(u)$ & Time at which node $u$ receives the message over tree $T$. \\
\hline$g_{T}(u)$ & Number of receiver nodes in the subtree rooted at $u$ in tree $T$ (including $u$ itself). \\
\hline$f_{T}(u)$ & Total delay in the subtree rooted at $u$ in tree $T$ (with $t_{T}(u)=0$ ). \\
\hline$h_{T}(u)$ & The delay from $v$ to its farthest descendant. \\
\hline$D, D_{\max }$ & Average and maximum node degree in the overlay. \\
\hline$L, L_{\max }$ & Average and maximum hop count of pairwise shortest paths in the overlay. \\
\hline & \\
\hline
\end{tabular}

\section{System Model ANd Problem Statement}

This section presents the formal statement of our multicast problems and the proof of their hardness, followed by a description of the routing model underlying our algorithm.

\section{A. Notation}

A summary of the notations used in this paper is given in Table I. Consider an overlay network interconnecting a large population of distributed hosts. The overlay is modeled by a graph $G=(V, E)$ in which each vertex $v \in V$ represents a host and each edge $(u, v) \in E$ represents a direct link between two connected hosts. Let $N=|V|$, and $w(u, v)$ be the length of edge $(u, v)$, which is the network delay between nodes $u$ and $v$ in our application. We assume each overlay node is connected to the underlying network, typically the Internet, via one link (nodes are assumed not multi-homed). The connection bandwidth of each node is a finite number, according to which we define $\Delta_{u}(z)$ as the time it takes for node $u$ to send $z$ units of data to the network. Since the time for node $u$ to process a message is much smaller than the time it takes to send out (possibly multiple copies of) the message to the network, we ignore the processing time at $u$ and let $\Delta_{u}(\cdot)$ be a function of the connection bandwidth of $u$ only. For example, for a host with a $10 \mathrm{Mbps}$ Internet connection and a message of $z=1$ bit, $\Delta_{u}(z)=10^{-7}$ seconds.

We capture the node degree-incurred delays (also referred to as nodal delays) in a multicast tree as 
follows. Let $T$ denote an arbitrary multicast tree rooted at a given source $s$ and reaching the receiver set $R \subseteq V$, and $d_{T}(u)$ be the number of outgoing links of $u$ in $T$. Once $s$ started distributing a message of size $z$ at time $0, t_{T}(v)$ is the time at which node $v$ receives the message over tree $T$. Assuming node $u$ is the parent of $v$ in $T, t_{T}(v)$ can be calculated using a new edge cost function $c_{T}(u, v)$ that we define as follows:

$$
\begin{aligned}
& c_{T}(u, v)=w(u, v)+\Delta_{u}(z) \times q_{u, T}(v) \\
& t_{T}(v)=t_{T}(u)+c_{T}(u, v), t_{T}(s)=0
\end{aligned}
$$

where $q_{u, T}(v)\left(1 \leq q_{u, T}(v) \leq d_{T}(u)\right)$ shows the turn of node $v$ among the $d_{T}(u)$ children of $u$ in $T$ that are waiting to receive a copy of the message.

Because we may not be able to explicitly dictate an order among the children of $u$ in $T$, we also take the expected delay observed at each child of $u$ into account. We define $\hat{c}_{T}(u, v)$ as in the following equation, by replacing $q_{u, T}(v)$ in Eq. (1) with $E\left[q_{u, T}(v)\right]$, the average of possible turns between 1 and $d_{T}(u)$ for a child.

$$
\begin{aligned}
& E\left[q_{u, T}(v)\right]=\frac{1}{d_{T}(u)} \sum_{i=1}^{d_{T}(u)} i=\left(d_{T}(u)+1\right) / 2 \\
& \hat{c}_{T}(u, v)=w(u, v)+z \times \Delta_{u}(z) \times \frac{d_{T}(u)+1}{2} \\
& \hat{t}_{T}(v)=\hat{t}_{T}(u)+\hat{c}_{T}(u, v), \hat{t}_{T}(s)=0 .
\end{aligned}
$$

\section{B. Formal Definition of the Problems and Proof of Hardness}

We consider two versions of the minimum delay multicast problem: (i) constructing a multicast tree in which the average delay (equivalently, the total delay) is minimized, and (ii) a tree in which the delay to the farthest node is minimized. We propose algorithms for both versions of the problem.

Our algorithms for case (i) do not require the encoding of the tree structure in full in the data; they do not need to specify a forwarding order for each intermediate tree node, and can work with $\hat{t}_{T}(v)$ values defined in Eq. (4). This is preferred, as it enables the use of mechanisms such as Bloom Filters for encoding the tree [15]. The algorithms for case (ii), on the other hand, have to specify a forwarding order for the children of each node (and therefore work with $t_{T}(v)$ values defined in Eq. (2)), since the maximum delay in a tree depends to a large extent on the ordering of each node's children.

Problem 1: Given a source node $s$ and a set of receivers $R \subseteq V$, construct a multicast tree $T$ such that $\sum_{u \in R} \hat{t}_{T}(u)$ is minimized. 


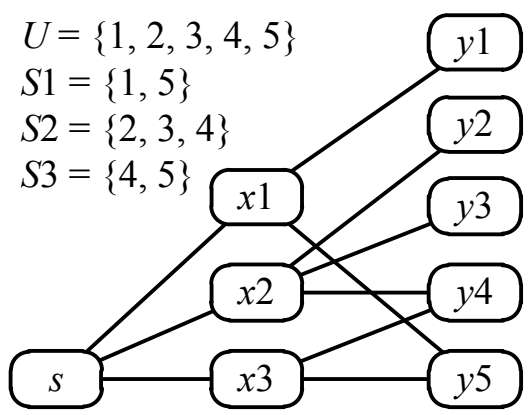

Fig. 1. Sample reduction from set cover to the min-delay multicast problem.

Problem 2: With the same inputs as in Problem 1, construct a multicast tree $T$ such that $\max _{u \in R} t_{T}(u)$ is minimized.

The total expected delay in a multicast tree, $\sum_{u \in R} \hat{t}_{T}(u)$, can be rewritten as the following for simplicity in the theorem below and our algorithms. Let us define an auxiliary cost function $f_{T}(u)$ for each node $u$ which is the total delay (in terms of $\hat{t}_{T}(\cdot)$ values) in the subtree rooted at $u$ in $T$, and let $g_{T}(u)$ be the number of receivers in this subtree. We have:

$$
\begin{aligned}
f_{T}(u)=\sum_{v \in \text { children }_{T}(u)}\left(f_{T}(v)+g_{T}(v) \hat{c}_{T}(u, v)\right) \\
\sum_{u \in R} \hat{t}_{T}(u)=f_{T}(s)=\sum_{v \in \text { children }_{T}(s)}\left(f_{T}(v)+g_{T}(v) w(s, v)\right)+ \\
|R| \times z \times \Delta_{s}(z) \times \frac{d_{T}(s)+1}{2} .
\end{aligned}
$$

Theorem 1 shows the NP-hardness as well as an inapproximability factor for Problems 1 and 2.

Theorem 1: Problems 1 and 2 are NP-hard, and also no polynomial time approximation algorithm for either of them can guarantee an approximation factor of $(1-\epsilon) \ln n$ for any $\epsilon>0$ (under the conventional assumptions for $\mathrm{P}$ and $\mathrm{NP}$ ).

Proof: The proof for both problems is based on reduction from the set cover problem. An instance $(U, S)$ of the set cover problem consists of a universe $U$ of elements $u_{j} \in U(1 \leq j \leq|U|)$ and a collection $S$ of subsets $S_{i} \subseteq U(1 \leq i \leq|S|)$ whose union is $U$. The goal is to find a minimum number of subsets whose union covers the entire $U$. We can transform an instance of the set cover problem to an instance of the overlay multicast problem as follows. Each subset $S_{i} \in S$ is represented by a node $x_{i}(1 \leq i \leq|S|)$ in the overlay, and each element $u \in U$ is represented by a node $y_{j}(1 \leq j \leq|U|)$. Figure 1 illustrates an example. An additional node $s$ is added to the tree as the source node. There is 
an edge from $s$ to all $x_{i}$, and an edge from $x_{i}$ to $y_{j}$ iff subset $S_{i}$ includes the element $u_{j}$. A sample instance of the set cover problem and the corresponding overlay are illustrated in Fig. 1. We also assign:

$$
\begin{aligned}
& R=\left\{y_{1}, \ldots, y_{|U|}\right\}, \Delta_{s}(z)=1, \Delta_{x_{i}}(z)=\Delta_{y_{j}}(z)=0, \\
& w\left(s, x_{i}\right)=w\left(x_{i}, y_{j}\right)=0(1 \leq i \leq|S|, 1 \leq j \leq|U|) .
\end{aligned}
$$

One can easily verify that given a multicast tree $T$ on this network that either minimizes $\sum_{u \in R} \hat{t}_{T}(u)$ or $\max _{u \in R} t_{T}(u)$, the set of nodes $x_{i}$ that are included in $T$ gives the solution to the set cover problem (consisting of $d_{T}(s)$ subsets). Hence, Problems 1 and 2 are NP-hard.

To establish the inapproximability bound, we first note that according to Eq. (5) the total and the maximum delay of a multicast tree $T$ on this network for distributing a unit-size message can be calculated as follows:

$$
\begin{aligned}
& \sum_{u \in R} \hat{t}_{T}(u)=f_{T}(s)=\frac{d_{T}(s)+1}{2} \times|U| \\
& \max _{u \in R} t_{T}(u)=\max _{v \in \operatorname{children}_{T}(s)} q_{s, T}(v)=d_{T}(s) .
\end{aligned}
$$

Suppose we are given an $\alpha$-approximation algorithm $(\alpha>1)$ for Problem 1, which can build a multicast tree $T$ in which the total distances to all nodes is at most $\alpha \times O P T$, where $O P T$ is the total distances in the optimal tree $T_{O P T}$. Hence, according to Eq. (6) we have:

$$
\begin{aligned}
& \sum_{u \in R} \hat{t}_{T}(u) \leq \alpha \times O P T \Rightarrow d_{T}(s)+1 \leq \alpha\left(d_{T_{O P T}}(s)+1\right) \\
& \Rightarrow d_{T}(s) \leq \alpha d_{T_{O P T}}(s) .
\end{aligned}
$$

Similarly, given an $\alpha$-approximation algorithm for Problem 2 and assuming $O P T$ is the maximum distance in the optimal tree $T_{O P T}$ for this problem, according to Eq. (7) we have:

$$
\max _{u \in R} t_{T}(u) \leq \alpha \times O P T \Rightarrow d_{T}(s) \leq \alpha d_{T_{O P T}}(s)
$$

Thus, in both cases we could find an $\alpha$-approximation algorithm for the set cover problem. On the other hand, given the well known $(1-\epsilon) \ln n$ inapproximability of the set cover problem in polynomial time [30], it is clear that Problems 1 and 2 cannot be approximated in polynomial time to within a factor of $(1-\epsilon) \ln n$ for any $\epsilon>0$, since otherwise a contradiction results. 


\section{Routing Model}

To enable a fully distributed routing scheme, overlay nodes need to generate and exchange periodic routing information such as measured distances to neighbors; in our case, also the announced $\Delta$ value of nodes. Link-state routing protocols, which are commonly used for overlay routing [12], [15]-[17], [19], [22], enable each node to know the full network topology by getting each node to broadcast the state of its neighboring links to the entire network. This scheme, however, has limited scalability due to its high overhead: $O\left(D^{2} N\right)$ per node ${ }^{1}$ where $D$ is the average degree in the overlay; each node generates a message of size $O(D)$ which traverses $O(|E|) \simeq O(D N)$ links. On the other hand, distance-vector routing used in [20], [21] incurs a lower overhead since each node announces to its neighbors only its shortest distance to other nodes. The corresponding overhead is on average $O(D N)$ per node-up to multiple orders of magnitude more scalable than link-state routing in well connected overlays. However, nodes will have no information about the paths other than the next hop and the total length. It is thus not possible for a source node to customize a multicast tree. Distance-vector routing can also suffer from routing loops and long convergence times.

Therefore, we adopt a modified version of distance-vector routing, where in addition to the shortest distance to each destination, a node also announces the path itself (in our case the nodes also announce their $\Delta_{u}(\cdot)$ values along with this information). This is similar to the technique employed in the BGP protocol [31] and is usually referred to as path-vector routing. This approach allows each node $u$ to construct a graph $G_{P V}^{u}=\left(V_{P V}^{u}, E_{P V}^{u}\right)$ where $V_{P V}^{u}=V$, and $E_{P V}^{u}$ consists of only a representative subset of $E$ for $u$ : the edges on the shortest path, as well as up to $d_{G}(u)-1$ alternative short paths, from $u$ to all destinations in the graph. This is because the information available at $u$ to construct $G_{u}^{\prime}$ consists of the shortest path from any of its $d_{G}(u)$ neighbors to all destinations in the graph. The overhead for exchanging path information is on average $O(D L N)$ per node, where $L$ is the average shortest-path length in the overlay. Note that $L$ (which is upper-bounded by the overlay diameter) is a small number in a well connected overlay [32], [33]; 5-10 in all of our experiments with different overlay models and $N=100, \ldots, 4000$ nodes. Consequently, we can inherit much of the scalability of distance-vector routing since the $O(D L N)$ overhead is much closer to that of distance-vector routing $(O(D N))$ as opposed to link-state routing $\left(O\left(D^{2} N\right)\right)$.

Moreover, path-vector routing does not suffer from routing loops and long convergence time as in the distance-vector approach, since the path info is given. Note that our multicast algorithms can indeed work

\footnotetext{
${ }^{1}$ These rough estimates are used for a first assessment only.
} 
on top of link-state routing protocols as well, in scenarios where the higher overhead is not a concern.

\section{Minimum-Delay Multicast Algorithms}

In this section, we first provide an overview of our minimum-delay multicast tree algorithms, and then present the details as well as the analysis of the algorithms.

\section{A. Overview of the Algorithms}

Our algorithms include two operation modes: MinSum for minimizing the expected total delay (Problem 1), and MinMax for minimizing the maximum delay in the tree (Problem 2). We refer to these algorithms as MSDOM and MMDOM (MinSum/MinMax Delay Overlay Multicast). These algorithms outperform the previous related approaches in both multicast tree efficiency and running time (and hence the supported scale), as analyzed in the next section. Nevertheless, to further extend the application of our work to larger overlays, we design an additional algorithm for each operation mode (MinSum/MinMax) that is optimized for speed. These algorithms make a slight sacrifice in tree efficiency while gaining orders of magnitude faster running times. They are particularly suitable for large overlays where our former algorithms (and the related previous approaches) cannot operate fast enough. We refer to the former (delay-efficient) algorithms as MSDOM/MMDOM-e, and to the latter (fast) version as MSDOM/MMDOM- $f$ algorithms.

Each overlay node that is to distribute a message runs the relevant version of the algorithm based on the target applications. The input to the algorithm consists of the state of the node's links to its neighbors, as well as the path vector information that the node has received from its neighbors. The resulting multicast tree is then encoded in the message. The intermediate overlay nodes are free of any additional computations or keeping any per-session multicast state information-they only forward the data to their neighbors according to the information embedded in the packets.

The multicast tree calculated by the source node can either be encoded in full in the message (with an overhead of $O(N)$ ), or be encoded as a digest, e.g., using fixed-size Bloom Filters that can significantly reduce the overhead with negligible false positives [15]. In this paper we only study the calculation of minimum-delay multicast trees, and the detailed encoding of the tree is out of the scope of the paper. Nevertheless, we highlight that the MSDOM algorithms do not need to specify the full tree structure for the intermediate forwarding nodes - they allow the use of Bloom Filter digests. The MMDOM algorithms, on the other hand, requires to signal the full tree structure (as in the similar work in [13]), because a forwarding order among the children of each node needs to be specified so that the forecasted maximum delay can be actually met in the network; see $q_{u, T}(v)$ in Eq. (1). 


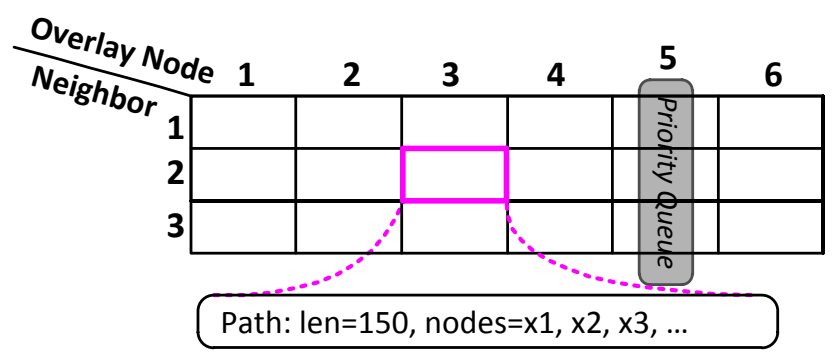

Fig. 2. Routing matrix at each node.

At a high level, the MSDOM-e algorithm gradually adds nodes to the tree according to the total cost of (i.e., delay increase for the whole tree by) any possible attachment of unattached nodes to the tree. The MMDOM-e algorithm repeatedly finds the receiver with the farthest distance (combined link and nodal delay) and attaches it to the tree, while carefully considering the change in all distances caused by attaching each new node. The MSDOM/MMDOM- $f$ algorithms take a different approach. They first build a regular shortest-path tree to the given set of receivers. Then, the tree is gradually refined by changing the route to some nodes, such that the total or the maximum delay is minimized.

\section{B. Detailed Routing Algorithms}

The main data structure used by our algorithms is a $k \times N$ routing matrix illustrated in Figure 2, where $k$ is the number of neighbors of the source node $s$ in the overlay $\left(k=d_{G}(s)\right)$, and $N$ is the total number of overlay nodes. Let us refer to overlay nodes $v_{1}, \ldots, v_{N}$ simply by their indices as nodes $1, \ldots, N$. An entry $(i, j)$ of the routing matrix of node $s$ represents the shortest path to overlay node $j$ through the $i$-th neighbor of $s$.

Each row $i$ of this matrix is maintained over time according to the path-vector information that the node receives from the corresponding neighbor, referred to as node $a_{i}$ in the following. A path-vector of the form $\left(\left\{x_{1}, \ldots, x_{e}, j\right\}, l\right)$ from neighbor $a_{i}$ indicates that the shortest path from node $a_{i}$ to node $j$ consists of the given $e+1$ hops and has a total length of $l$. This will update the $(i, j)$ entry of the routing matrix as $\left(\left\{a_{i}, x_{1}, \ldots, x_{e}, j\right\}, l+\tilde{w}\left(s, a_{i}\right)\right)$, where $\tilde{w}\left(s, a_{i}\right)$ is the distance to neighbor $a_{i}$ measured at $s$. Moreover, a priority queue (min-heap) is maintained per each column of the routing matrix, which can immediately return the best neighbor for reaching each destination, i.e., the row with minimum path length. We analyze the complexity of maintaining the routing matrix shortly.

Our multicast algorithms are specified in Figures 3 and 4. In the MSDOM-e algorithm, we start from the source $s$ and build the multicast tree by incrementally adding nodes according to their distances to the 


\section{Minimum End-to-end Delay Overlay Multicast}

\section{MSDOM_e( $)^{\dagger}$}

1. $S=V-\{s\} ; T=$ BuildEmptyTree $(s)$

2. $t[i=1, \ldots, N]=\infty ; t[s]=0$

3. while $R \neq \emptyset$ do

4. $\quad$ cost $=\emptyset ;$ prev $=\emptyset$

5. $\quad$ for $v$ in $S$ do

6. $\quad \operatorname{cost}^{\prime}=\emptyset$

7. for $u$ in neighbors $\left(v, G_{P V}\right)$ do

8. $\quad$ if $u \notin T$ then continue

9. $\quad / / C o s t$ of attaching $v$ to tree $T$ through $u$ :

10. $\quad \operatorname{cost}^{\prime}[u]=t[v]+\tilde{w}(u, v)+\left(d_{T}(u)+2\right) / 2 \times \Delta_{u}(z)$

11. $\operatorname{cost}^{\prime}[u]+=1 / 2 \times \Delta_{u}(z) \times\left(\right.$ SubTreeSize $\left._{T}(u)-1\right)$

12. $u^{*}=\operatorname{argmin}\left(\cos t^{\prime}\right)$

13. if $u^{*}==N U L L$ then continue

14. $\operatorname{cost}[v]=\cos t^{\prime}\left[u^{*}\right]$

15. $\operatorname{prev}[v]=u^{*}$

16. $v^{*}=\operatorname{argmin}($ cost $) ; u^{*}=\operatorname{prev}\left[v^{*}\right]$

17. AttachToTree $\left(T, v^{*}, u^{*}\right)$

18. S.remove $\left(v^{*}\right)$

19. if $v^{*} \in R$ then R.remove $\left(v^{*}\right)$

20. Update_t $t\left(T, u^{*}, t[]\right)$

21. $\operatorname{CleanUp}(T, s)$

22. return $T$

MMDOM_e ()$^{\dagger}$

1. $T=$ BuildEmptyTree $(s) ; t[i=1, \ldots, N]=\infty ; t[s]=0$

2. while $R \neq \emptyset$ do

3. for $u$ in $V$ do

4. $\quad \operatorname{prev}[u]=-1$

5. $\quad \operatorname{dist}[u]=t[u]+d_{T}(u) \times \Delta_{u}(z)$

6. $S=V$

7. while $S \neq \emptyset$ do

8. $u^{*}=\operatorname{argmin}_{u \in S}(\operatorname{dist}[u])$

9. S.remove $\left(u^{*}\right)$

10. for $v$ in neighbors $\left(u^{*}, G_{P V}\right)$ do

11. $\quad$ if $v \in T$ then continue

12. $\quad d=\operatorname{dist}\left[u^{*}\right]+\Delta_{u^{*}}(z)+\tilde{w}\left(u^{*}, v\right)$

13. $\quad$ if $d<\operatorname{dist}[v]$ then

14. $\operatorname{dist}[v]=d$

15. $\quad \operatorname{prev}[v]=u^{*}$

16. // Attach the farthest node to tree $T$ :

17. $v^{*}=\operatorname{argmax}_{v \in R \backslash T}(\operatorname{dist}[v])$

18. $H=$ List containing hops of path to $v^{*}$ according to prev[]

19. for $v$ from H.FirstNodeNotInTree(T) to H.last() do

20. $u=\operatorname{prev}[v] \quad / /$ Parent of the to-be-attached node $v$ in $T$

21. AttachToTree $(T, v, u)$

22. $\quad t[v]=\operatorname{dist}[v]$

23. $\quad$ if $v \in R$ then $\operatorname{Rremove}(v)$

24. return $T$

$\dagger$ Variables used in the code are described in Table I.

Fig. 3. The proposed overlay multicast algorithm. 
current tree. More specifically, for each potential attachment $(u, v)$ to the tree $T$ where $u \in T$ and $v \notin T$ (Lines 5 to 8 ), we find the cost as the increase in the expected total delay caused by this attachment (Lines 10 and 11). This delay consists of the expected delay to node $v$ itself, as well as the expected delay to be suffered from by other descendants of $u$ since the degree of $u$ is going to increase (see Eqs. 3 and 4). Having applied the minimum-cost attachment $\left(u^{*}, v^{*}\right)$ (Lines 16 to 18), we update the current distance value (array $t$ ) of all affected nodes (Line 20), i.e., the children and all further descendants of node $u^{*}$. Finally, we cleanup the tree (Line 21) by keeping only the links that reach some receiver nodes.

The MMDOM-e algorithm repeatedly runs our modified version of the Dijkstra algorithm to find the farthest node from the current tree $T$. This modified shortest-path algorithm (Lines 3 to 15) can start from multiple nodes $(\forall u \in T$; see the initialization of dist [] in Line 5), considers the current degree of nodes in the existing tree (Line 5), and expands based on aggregated link and nodal delays (Line 12). Having found the farthest node $v^{*}$ from the current tree $T, v^{*}$ as well as its predecessors (list $H$ ) on the path starting from some node in $T$ are added to the tree. Note that after this addition, the degree of a number of nodes changes, making the recently calculated distances no longer accurate. The next farthest node is thus searched for again in the next iteration.

The running time of the MinSum and MinMax algorithms presented so far, as we analyze shortly, is $O\left(N^{2} D_{P V}\right)$ and $O\left(N^{3}\right)$, respectively, where $D_{P V}$ is the average degree in the path-vector based view of the overlay graph. This running time may not be efficient enough for large overlays, as we analyze in the next section. We therefore develop additional algorithms, MSDOM/MMDOM- $f$, which are optimized for speed.

In these algorithms, which are illustrated in Figure 4, we first calculate the regular shortest-path tree from $s$ to the receivers. For this purpose, in the source node's routing table we visit the priority queue corresponding to each destination, and take the path pointed to by the queue head. The union of these paths forms the initial routing tree $T$. An example is given in Figure 5 to illustrate the main operations of these algorithms. The solid lines in this figure represent the shortest-path tree created so far. This tree is then refined according to the given objective: minimizing the total delay or the maximum delay.

In the MSDOM- $f$ algorithm, the tree is refined in a top-down manner from the root downwards. For each node $u$, we look at each of its children $v$ and consider routing to it through an alternative route (Lines 5 and 6). The alternatives for a node $v$ are to route to $v$ through any other of the $d_{G}(s)$ neighbors of the root $s$ than the one currently used to reach $v$. Given the path vector information available at $s$, we evaluate the possible alternative routes, and change the current route to $v$ if necessary (Lines 7 to 10). To refine the route to the children of node $u$, we first consider those children that have the highest 


\section{Minimum End-to-end Delay Overlay Multicast}

MSDOM_MMDOM_f $(M[][], Q[]$, mode $)$

// $M[][]$ : Routing matrix.

// $Q[]$ : Priority queues on the columns of $M$.

// mode: MinSum or MinMax.

// For other variables used in the code see Table I.

1. $T=$ BuildEmptyTree $(s)$

2. for $r$ in $R[]$ do

3. $i^{*}=Q[r] \cdot \operatorname{Min}()$

4. $P=M\left[i^{*}\right][r]$

5. T.InsertPath $(P)$

6. RefineTree $[$ mode $](T, s, t[])$

7. return $T$

RefineTree[MinSum] $(T, u, t[])$

1. if $u . I s L e a f()$ then

2. return

3. for $v$ in u.children () in decreasing order of $g_{T}(C[i])$ do

4. $t^{\prime}[]=\operatorname{EmptyArray}\left(d_{G}(\right.$ T.root $\left.)\right)$

5. for $a$ in T.root.neighbors () do

6. $\quad t^{\prime}[a]=$ SavingByRouteChange $(T, v, a)$

7. $a^{*}=\operatorname{argmax}\left\{t^{\prime}[]\right\}$

8. if $t^{\prime}\left[a^{*}\right]>0$ then

9. T.DeleteRoute( $v) \quad / / \operatorname{detach} v$ and its subtree

10. T.InsertRoute $\left(v, a^{*}\right)$

11. Sort $C[]$ in decreasing $g_{T}(C[i])$

12. for $v$ in u.children () in decreasing order of $g_{T}(C[i])$ do

13. RefineTree $(T, v, t)$

RefineTree[MinMax] $(T, u, t[])$

1. T.FixForMinMax ()

2. for $i=1$ to MAX_REFININGS do

3. $v=$ T.FarthestLeaf ()

4. $t^{\prime}[]=\operatorname{EmptyArray}\left(d_{G}(\right.$ T.root $\left.)\right)$

5. for $a$ in T.root.neighbors() do

6. $\quad t^{\prime}[a]=$ SavingByRouteChange $(T, v, a)$

7. $a^{*}=\operatorname{argmax}\left\{t^{\prime}[]\right\}$

8. if $t^{\prime}\left[a^{*}\right] \leq 0$ then //no more refinings

9. break

10. T.DeleteRoute $(v) \quad / / \operatorname{detach} v$ and its subtree

11. T.InsertRoute $\left(v, a^{*}\right)$

12. while $v \neq$ T.root do

13. $v=$ v.parent

14. v.FixChildrenForMinMax ()

15. T.FixNodesDS()

Fig. 4. The proposed routing algorithm. 


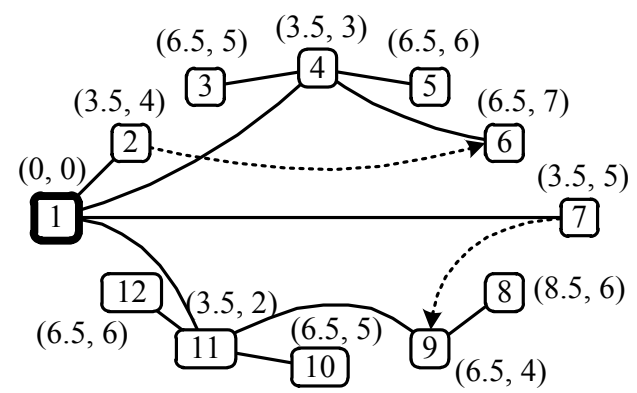

Fig. 5. An example overlay multicast tree. Solid lines represent the regular shortest-path tree. Dashed lines represent two possible overlay edges to refine the tree. Numbers next to each node show the time at which the node receives the message: expected time (Eq. (4); used in MSDOM) and the exact time (Eq. (2); used in MMDOM), respectively. $z, \Delta_{u}(z)$ for all nodes, and $w(u, v)$ for all solid-line links are equal to $1 . w(u, v)$ for the two dashed links equals 1.5 . The source is node 1 , and the receivers are 2 to 12 .

number of receivers in their subtrees (the sort operation in Line 2), since any saving in the delay to those children will likely yield a higher overall saving. Once finished relaxing the degree of node $u$, the algorithm proceeds with the next level which is refining the subtree of each child of $u$.

In the example illustrated in Figure 5, a sample refining step is to change the route to node 9 from $(1 \rightarrow 11 \rightarrow 9)$ to $(1 \rightarrow 7 \rightarrow 9)$. Then, according to the given link and node delays in Figure 5 , the expected message delivery time is reduced from 6.5 to 6 for node 9 , and from 8.5 to 8 for node 8 (see Eq. (4)). Moreover, since the number of children of node 11 is reduced by one, the expected delivery time to nodes 10 and 12 will also reduce from 6.5 to 6 . That is, we can achieve a total saving of 2 by applying the above route change.

For the MMDOM- $f$ algorithm, we first note that the maximum delay in the subtree rooted at a node $u$ can vary much based on the ordering of $u$ 's children to receive the message (see $q_{u, T}(v)$ in Eq. 1). Thus, we need to obtain an optimal ordering for the children of $u$ in the tree, and to mandate this ordering for the physical node $u$ to follow. Denoting by $h_{T}(v)$ the delay from $v$ to its farthest descendant, the optimal ordering of $u$ 's children for minimizing the maximum delay corresponds to sorting the children in decreasing order of their $h_{T}(v)$ values. This is done for all nodes of the tree in function T.FixForMinMax (Line 1).

The numbers shown next to the nodes in Figure 5 as the exact delivery time are calculated according to this optimal ordering. For example the children of node 11 are ordered as $(9,10,12)$ since the farthest descendant of node 9 is at distance $h_{T}$ (node 9$)=2$, while this value is 0 for nodes 10 and 12; similarly, the children of the root are ordered as $(11,4,2,7)$.

Then, in each iteration the algorithm picks the node with maximum delay (Line 3), and tries to find an 
alternative shorter route to the node (Lines 5 and 6). In the example illustrated in Figure 5, this operation includes changing the route to node 6 from $(1 \rightarrow 4 \rightarrow 6)$ to $(1 \rightarrow 2 \rightarrow 6)$. According to Eq. (2), this update reduces the delivery time to node 6 from 7 to 6.5 .

The algorithm terminates if the tree reaches a state where the distance to the farthest node cannot be shortened (Line 8), or if the total number of refining steps reaches a bound (MAX_REFININGS in the code). We set this bound to $N / \log N$ ) to keep the worst-case running time to $O\left(N^{2}\right.$ ) (analyzed shortly), although in our experiments the algorithm has terminated much earlier than this bound. To enable efficient reordering of each node's children and retrieval of the farthest node in the tree, at each node $v$ we maintain certain information including $t_{T}(v)$ and $h_{T}(v)$. After each modification in the tree, the information maintained at up to $O(N)$ nodes may need to be updated. For example, after moving node $v$ from its old parent $u$ to a new parent $u^{\prime}$, the delay to each descendant of $v$, the correct ordering of the children of $u^{\prime}$ and those of $u^{\prime}$ 's ancestors, and accordingly the delay to each descendant of $u^{\prime}$ and $u$ 's ancestors needs to be updated. Therefore, we simply update the data structures for all nodes of the tree in $O(N)$ time (Line 15 of the pseudocode).

\section{Analysis}

The proposed algorithms need $O\left(N d_{G}(u) L_{\max }\right)$ of memory space to maintain the required data structures at an overlay node $u$ (see Figure 2), where $L_{\max }$ is the maximum hop count in a shortest path (i.e., the hop count of the overlay diameter). A node also maintains a path-vector based view of the overlay graph, which requires $O\left(N D_{P V}\right)$ memory. Moreover, while calculating a multicast tree, the source node needs to keep only $O(1)$ information per each tree node, hence a total of $O(N)$ memory is needed. Therefore, the total memory needed by the nodes is on average $O\left(N D L_{\max }\right)$.

Upon receiving a path vector of size $O\left(N L_{\max }\right)$, a node $u$ updates the corresponding row of its routing matrix. At most $N$ entries are updated, taking a total time of $O\left(\left(L_{\max }+\log d_{T}(u)\right) \times N\right)$ to write the new paths in the matrix and to update the priority queues of the corresponding columns.

The MSDOM-e algorithm repeatedly evaluates all possible new attachments to the current tree, which takes $O\left(N D_{P V}\right)$ time. The best attachment is then applied and all the distances (array $t$ ) are updated in a total time of $O(N)$. This is repeated $O(N)$ times, leading to a total running time of $O\left(N^{2} D_{P V}\right)$. In MMDOM-e, we repeat our modified version of the Dijkstra algorithm $|R|=O(N)$ times, each of which run in $O\left(N D_{P V}\right)$. The total running time of this algorithm is therefore $O\left(N^{2} D_{P V}\right)$. The MSDOM/MMDOM- $f$ algorithms, in both modes, first builds an initial shortest-path tree which requires up to $O\left(N L_{\max }\right)$ time, given the priority queues to return the shortest routes in $O(1)$ (see function 
$M S D O M \_M M D O M \_$). In the MinSum mode, for each node $u$ the algorithm takes $O\left(d_{T}(u) \log d_{T}(u)\right)$ time to sort its children in Lines 3 and 12 of function RefineTree[MinSum]. Then, the following operations are performed per each child of $u$ (i.e., $O\left(d_{T}(u)\right)$ times): $O\left(d_{T}(s) L_{\max }\right)$ in Lines 5 and 6 to find the saving with any of the $d_{T}(s)$ alternative routes, and $O\left(L_{\max }\right)$ in Lines 9 and 10 to modify the tree. Finally, the same operation is repeated for each child of $u$. Let us assume $\log d_{T}(u)<d_{T}(s)$ for any node $u$. Hence, the total time taken by function RefineTree[MinSum] for each node $u$ is given as:

$$
\begin{aligned}
\mathbb{T}(u) & =O\left(d_{T}(u) d_{T}(s) L_{\text {max }}\right)+\sum_{v \in \text { children }_{T}(u)} \mathbb{T}(v) \\
\Rightarrow & \mathbb{T}(s)=O\left(N d_{T}(s) L_{\text {max }}\right)
\end{aligned}
$$

since $\sum_{u \in T} d_{T}(u)=O(N)$ in a tree. The complexity of the MSDOM algorithm is therefore $O\left(N L_{\max }\right)+$ $O\left(N d_{T}(s) L_{\max }\right)=O\left(N d_{T}(s) L_{\max }\right)$.

In the MinMax mode, our algorithm first fixes the ordering of each node's children in Line 1 of function RefineTree[MinMax]. This is done by sorting the children of each node, which takes a total time of $O\left(\sum_{u \in T} d_{T}(u) \log d_{T}(u)\right)=O(N \log N)$. Then, in each iteration the farthest node is picked in $O(1)$ (maintained by Lines 1 and 15), and $O\left(d_{T}(s) L_{\max }\right)$ time is taken in Lines 5 and 6 to analyze the alternative routes to the node. Changing a route in the multicast tree and performing the corresponding updates (Lines 10-14) take a total of at most $O\left(L_{\max }\right)+O(N \log N)$ time, respectively. Finally, updating the data structures associated with the tree in Line 15 takes $O(N)$ time. Thus, each iteration takes a total of $O(N \log N)$ time. The algorithm continues until no better route is found to the current farthest node, or until the number of refining steps reaches MAX_REFININGS. By bounding this value to $N / \log N$, the total running time of MMDOM- $f$ is bounded by $O\left(N^{2}\right)$; though we also note that the number of iterations taken in our experiments has been far less than $N / \log N$.

\section{Evaluation}

We evaluate the performance of our algorithms on hundreds of overlay networks built on top of two different real-world Internet delay datasets, and according to three different overlay graph models. Our evaluation setup and the obtained results are presented in this section.

\section{A. Setup}

To capture the actual delay between hosts in the Internet, which is a key factor determining the effectiveness of any overlay multicast algorithm, we use the data from two different measurements: the 
Delay Space Synthesizer (DS ${ }^{2}$ ) project [34] which provides the pairwise delay between about 4000 hosts, and the Meridian project [35] containing the delay between 2500 hosts. We sometimes need to downsample the set of 4000 (or 2500) nodes to $N<4000$ nodes in our experiments. To ensure having a representative subset, we use a variant of the $k$-means clustering algorithm [36] that takes the best $N$ center points among the original 4000 (or 2500); it minimizes the sum of the square of distance between each original point and its closest center point.

On top of these datasets, we create overlay networks based on three different overlay graph models: small world [37], random [38], and power law [39]. In small-world networks, links are more likely to exist between neighbor nodes than distant nodes [37]. These networks are commonly observed in social and P2P networks [40], [41]; they have also yielded the smallest shortest-path length between nodes in our experiments. We generate these networks by connecting each pair of nodes $(u, v)$ with probability $\alpha$ distance $(u, v)^{-1}$, where the coefficient $\alpha$ is set according to the desired average node degree $D$ in the overlay. On the other hand, random networks are simpler in which all edges of different lengths are treated similarly. Specifically, we generate random networks according to the Erdos-Renyi model [38] where each pair of nodes $(u, v)$ exists with probability $p$, which we set according to the desired average degree $D$. In a power-law network (also called scale-free network), such as the worldwide web, nodes that are popular are more likely to receive new links; the network therefore has a power-law degree distribution [39]. We generate these networks following the Barabasi-Albert model [39], where a new node $x$ connects to an existing node $u$ with probability $\alpha d_{G}(u) / \sum_{v \in V} d_{G}(v)$ ( $\alpha$ determining the average degree). We conduct a comprehensive evaluation study by running experiments on these diverse network models.

The parameters that we vary in our evaluations include the overlay size $(N)$, average node degree $(D)$, number of receivers $(|R|)$, and nodal delays (including their dynamics). Specifically, to account for different node-incurred delays for a message, we define $\bar{\Delta}$ as the average time that it takes for a node to send out each copy of the message. To get a sense of various combinations of node bandwidth/workload as well as message size (which together govern the nodal delay), we consider a diverse range of values for $\bar{\Delta}$ from $10 \mathrm{~ms}$ to $1000 \mathrm{~ms}$, assumed the same $( \pm 50 \%)$ for all nodes. For example, $\bar{\Delta}=10 \mathrm{~ms}$ can represent the case where each node has a $10 \mathrm{Mbps}$ Internet connection and is handling 10 concurrent multicast sessions on average, and the message is a $10 \mathrm{Kbits}(1.2 \mathrm{~KB}) \mathrm{IP}$ packet; we also analyze the dynamics of nodal delays in our evaluations. Furthermore, to consider the delivery of a continuous flow of data, we note that each node would send the data in chunks to its children in the multicast tree. Assuming TCP transport, to maximize the throughput the node sends a continuous chunk of up to a full 
TCP window to its first child, then to the second child, and so on. The message size $z$ can therefore be assumed up to the maximum window size, e.g., $128 \mathrm{~KB}$ (1 Mbits) as it is the default value in most Linux distributions. Thus, $\bar{\Delta}=1000 \mathrm{~ms}$ can represent the multicast of a continuous flow on the aforementioned network.

Given an overlay network of $N$ nodes and a number of receivers $|R|$, in each run we select a sender and $|R|$ receivers at random from $[1, N]$. We then generate multicast trees using ours as well as previous approaches (described shortly) on the same input: $G_{P V}$ view of the overlay, $s$ and $R$. We repeat each experiment 100 times, comprising 10 different overlays and 10 different sender/receivers selection on each overlay, and we measure the average delay and running time obtained in the 100 cases. Finally, since in the MinSum algorithms a source does not transmit the full (ordered) tree structure (see Section III), we simulate this lack of knowledge by shuffling the children of each node in the created tree before evaluating the average delay to the receivers.

\section{B. Results}

We evaluate the MSDOM algorithms by comparing the achieved average delay with the average delay in the shortest-path tree (SPT) for the same overlay and receiver set. We also compare these results with the delay achieved by the algorithm in [27], which builds a MinSum-delay multicast tree with bounded degrees - the closest work in the literature to our MinSum algorithms, to the best of our knowledge. We run a binary search to find the best degree bound value for this algorithm, though in our running time measurements presented shortly we only measure the time taken by one run of the algorithm (not the multiple times done in the binary search). This algorithm is labeled as "MLRS" in the following figures (taken from the names of the authors).

We analyze the MMDOM algorithm by comparing the maximum delay in our tree with the maximum delay in the SPT as well as the tree created by the algorithm in [13] for the same overlay and receiver set. As discussed in Section II, the algorithm in [13] is the only previous study on minimizing the overall delay (including link- and node-incurred delays) in a multicast tree, to the best of our knowledge. We only consider the heuristic algorithm proposed by the authors, since it outperforms the proposed alternative approximation algorithm in both tree-efficiency and running time [13]. We also note that this work only addresses the MinMax version of our problem. This algorithm is label as "BLS" in the following figures.

We first evaluate the algorithms on overlays of different sizes, and present the results in Figure 6. We also report the time taken by these algorithms in Figure 7. Our experiments are run on a commodity PC with Intel Core i5 $2.67 \mathrm{GHz}$ CPU with $8 \mathrm{~GB}$ of memory. The following experiment (Figures 6 and 7) 


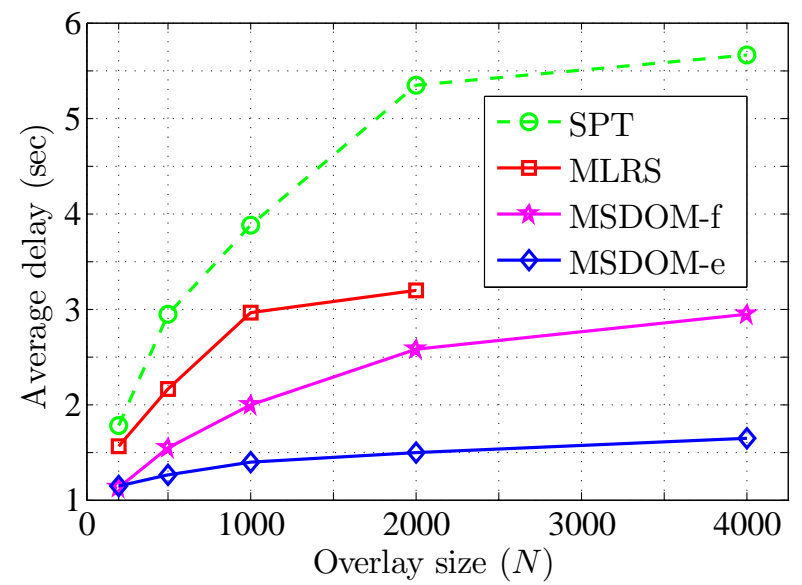

(a) MinSum-delay trees.

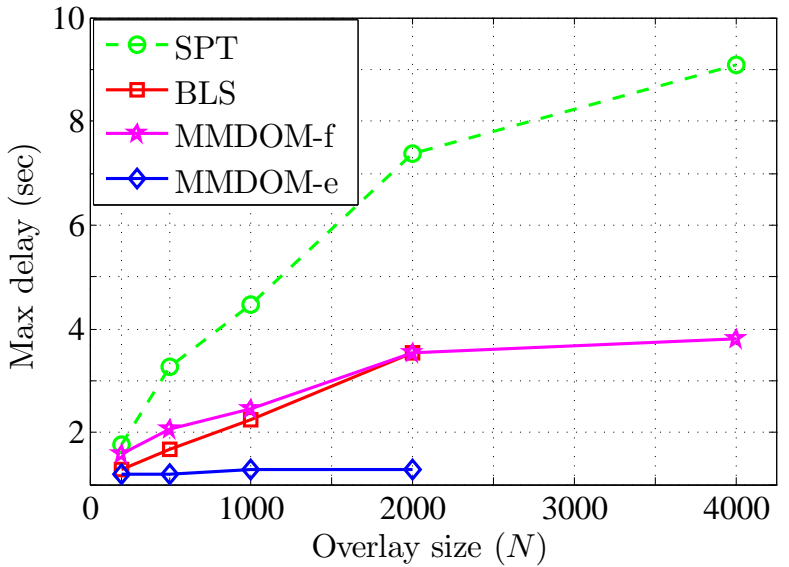

(b) MinMax-delay trees.

Fig. 6. Average and maximum delay of multicast trees created by different algorithms as well as their running times.

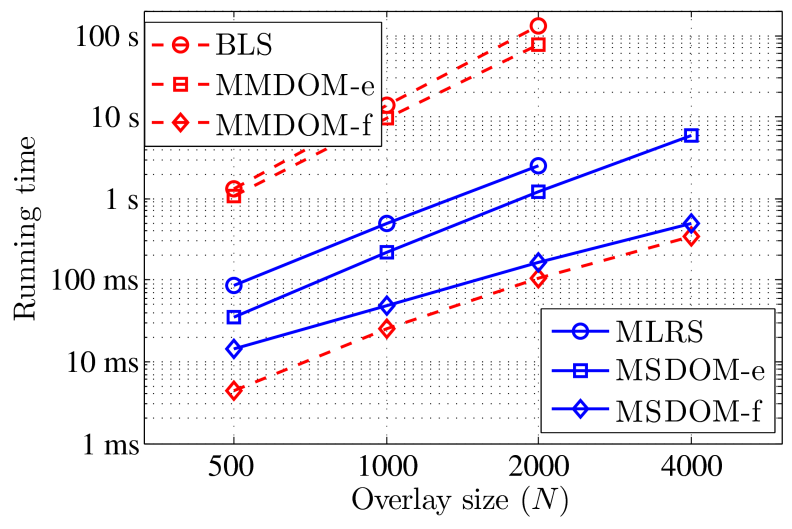

Fig. 7. The running time of the algorithms in Figure 6.

is conducted on the $\mathrm{DS}^{2}$ dataset, using the small-world overlay mode, with $R=N-1, D=N / 10$ and $\bar{\Delta}=100 \mathrm{~ms}$. The cases with $N=4000$ are skipped for MLRS, BLS, and MMDOM-e algorithms for their running times. Also, we do not report a running time for SPT in Figure 7 as it takes a very short time given the up-to-date routing table-only merging the shortest paths pointed to by priority-queue heads (see Figure 2). The routing table in turn is updated by a different mechanism, upon a route update from a neighbor, which takes a few milliseconds and then provides the underlying data for all of the evaluated algorithms. Nevertheless, to get a sense of the SPT running time independent of the routing mechanism, we consider the case that the routing table is maintained plainly without the priority queues. Thus, in order for a source node to find the best path to each receiver, it needs to search among the shortest paths announced by its neighbors, i.e., $O\left(|R| \times d_{G}(s)\right)$ for node $s$. This procedure takes 7,37 
and $154 \mathrm{~ms}$ on average for overlays of size $N=1000,2000$, and 4000, respectively.

It is noteworthy that one might add the running time to the delay achieved by the tree, since a message is not sent out until the tree calculation is done. However, we also note that each calculated tree is typically expected to be used for a number of messages. For instance, back to the example of the dynamic online agent (the source) moving across the area-of-interest of others (the receivers) in a virtual environment, we can realistically expect that the receiver group changes at a quite slower pace (in the order of seconds) than the rate of disseminating messages (several per second). We therefore separately report both times, tree delay and running time.

In Figure 6, we first observe that the commonly used shortest-path trees, despite being fast to build, suffer from large average and maximum delays as they are unaware of nodal delays. We also observe in Figure 6(a) that both of our algorithms outperform the previous related work MLRS [27]. MSDOM- $e$ trees can provide an average delay as low as half the MLRS ones, while also being created multiple times faster (note the log-log scale in Figure 7). Our fast algorithm MSDOM- $f$, is several times faster (taking $490 \mathrm{~ms}$ for $N=4000$ ) while still providing better average delays than MLRS.

Similarly for the MinMax version in Figure 6(b), we observe that MMDOM-e yields the smallest farthest-node delays $(<1.3 \mathrm{~s})$, significantly shorter than that of the related previous work BLS [13] which is up to $4 \mathrm{~s}$-between $8 \%$ to $64 \%$ less. We also subtly note that in a few cases the maximum delay achieved by MMDOM- $f$ is even slightly better that the average delay achieved by MSDOM- $f$, which is because the MinMax algorithms have the advantage of transmitting the ordered tree structure (as they have to) while the MinSum ones do not (see Section III). Furthermore, we observe in Figure 6(b) that the maximum delay achieved by the MMDOM- $f$ algorithm and the one by BLS are relatively close, with BLS being slightly better in some cases. On the other hand, the running time of MMDOM- $f$ is 2 to 3 orders of magnitude smaller (from 1 to $340 \mathrm{~ms}$ ). Therefore, with the MMDOM-e algorithm for overlay sizes of up to hundreds, and MMDOM- $f$ for larger overlays, we can create MinMax-delay multicast trees with much smaller delays (less than half) and/or faster running times (orders of magnitude) than the alternative approaches.

Next, we analyze our algorithms with different levels of network connectivity, defined by different values of the average node degree $D$. Figure 8 shows the average and the maximum delay in overlays of size $N=1000$ nodes. In this experiment, $|R|=999, \bar{\Delta}=100 \mathrm{~ms}$, and the overlays are built on the $\mathrm{DS}^{2}$ dataset and using the small-world model. As expected, the regular shortest-path tree that ignores node degrees results in an increasingly high delay as network connectivity increases in Figures 8(a) and 8(b). This is because shortest paths tend to be formed within very few hops of the source as the overlay 


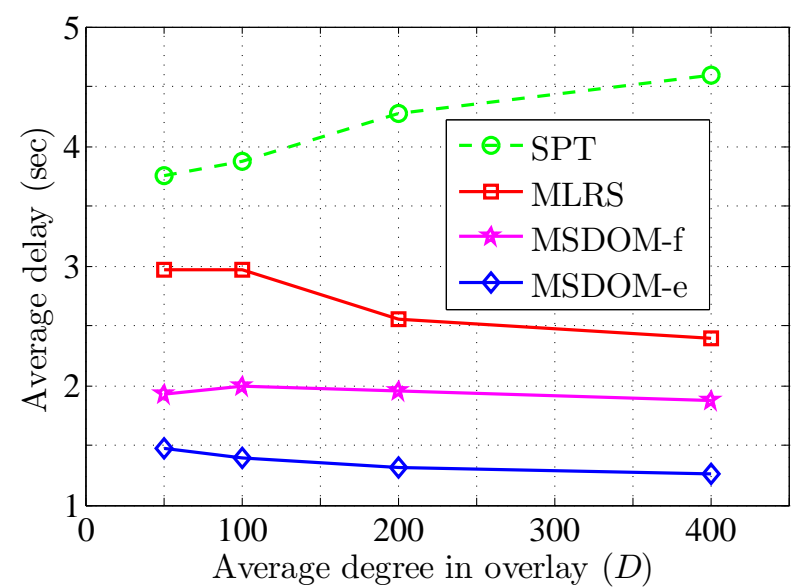

(a) MinSum-delay trees.

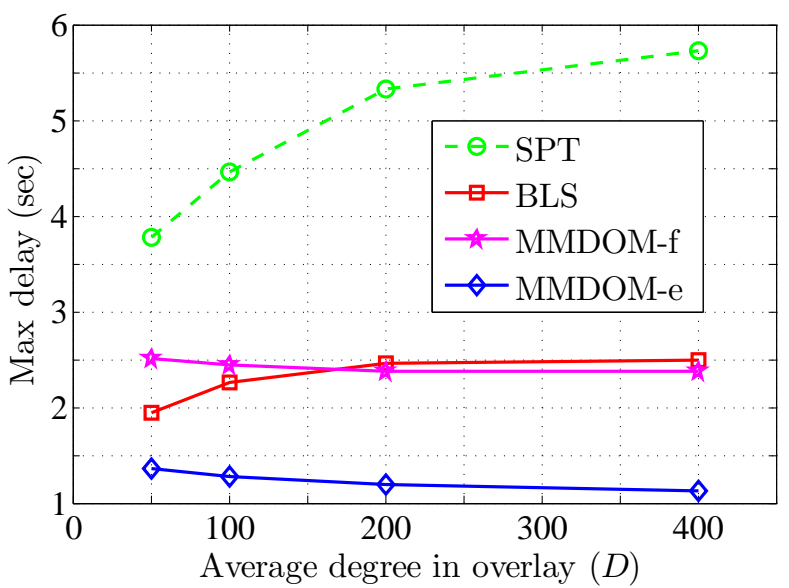

(b) MinMax-delay trees.

Fig. 8. Average and maximum delay of multicast trees for different levels of overlay connectivity. $N=1000$ and $|R|=999$ in the experiments.

gets denser. This yields high node degrees in the multicast tree, and accordingly high delays incurred at overlay nodes. On the other hand, the delays in multicast trees created by our algorithms are up to several times smaller. In addition, both MSDOM-e and MMDOM-e algorithms achieve a delay of about half the related works (BLS/MLRS). Moreover, MSDOM- $f$ trees provide an average delay between 20\% to $35 \%$ smaller than MLRS trees, while also being created 5 to 20 times faster. For the MinMax version, similar to the previous experiment, the farthest-node delay in MMDOM- $f$ and BLS trees are close, while the former one is created in 4-65 ms in this experiment and the latter in 13-15 seconds.

We continue our experiments by varying the number of receivers $|R|$. The other configurations remain the same, and $D=N / 10$. As plotted in Figure 9, the same trend as in the previous experiment is observed among the achieved delay and running time of algorithms. Moreover, we analyze the impact of $\bar{\Delta}$, the average nodal delay for each copy of the message, when varying in the range 10-1000 ms as described earlier. For the MinSum version (Figure 10(a)), we observe that a similar superiority among the trees made by different algorithms holds as in previous experiments, with roughly the same ratio among them for different $\bar{\Delta}$ values, e.g., MSDOM-e trees have an average delay between $43 \%$ to $54 \%$ less than MLRS trees. In the MinMax version, this trend is more or less the same except for small $\bar{\Delta}$ values, given that the dominant factor in the farthest-node delay becomes the longest shortest-path distance (i.e., the diameter) as the nodal delay approaches small values (particularly $10 \mathrm{~ms}$ ). This effect is not as bold in the MinSum case since it deals with the average shortest-path distance which is been several times smaller than the maximum in our experiments. We also see in Figure 10(b) that the MMDOM- $e$ algorithm yields 


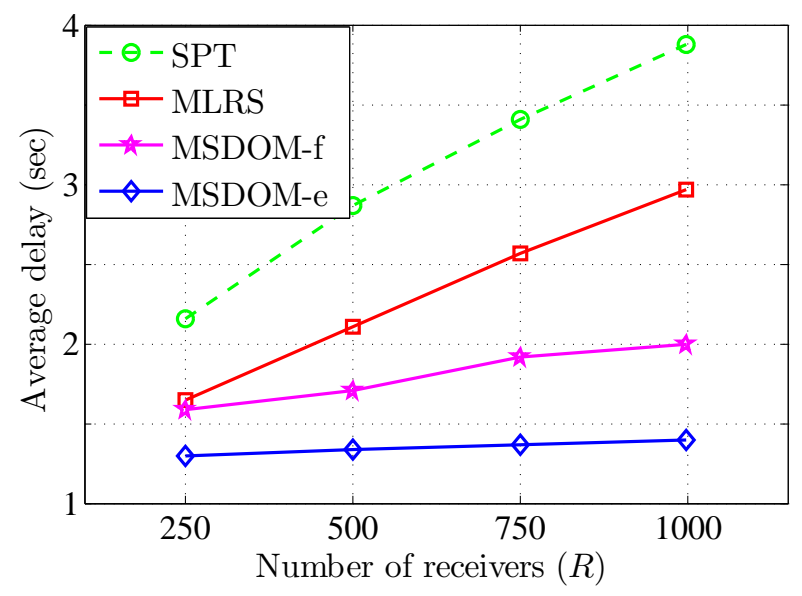

(a) MinSum-delay trees.

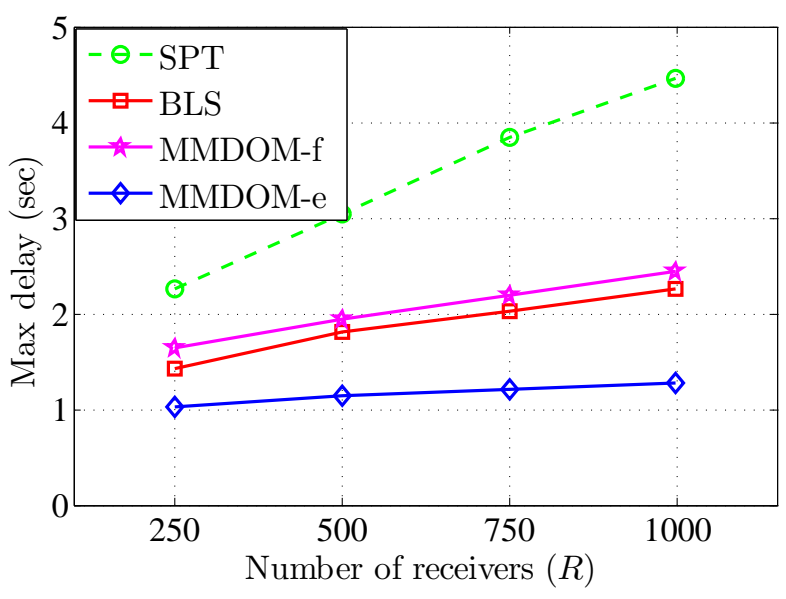

(b) MinMax-delay trees.

Fig. 9. Average and maximum delay of multicast trees for different number of receivers.

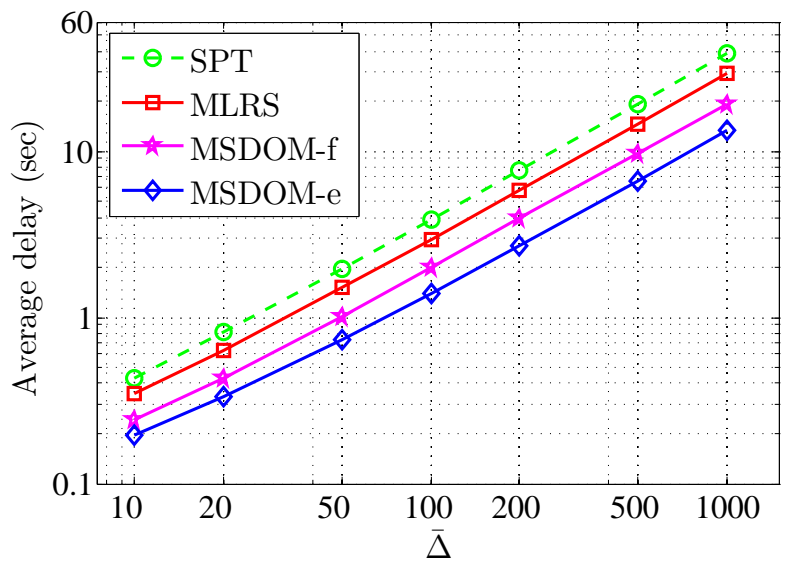

(a) MinSum-delay trees.

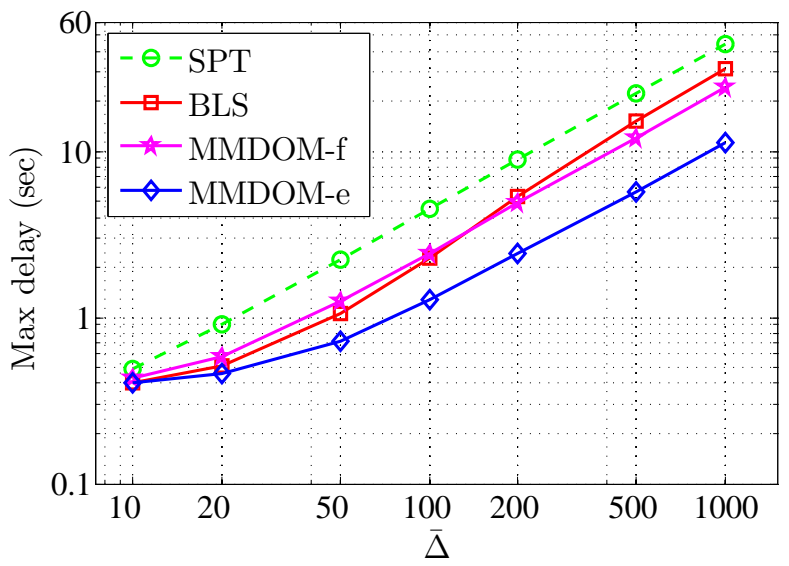

(b) MinMax-delay trees.

Fig. 10. Average and maximum delay of multicast trees for different values of $\bar{\Delta}$.

the smallest delays (up to $60 \%$ less than BLS), while still running slightly faster than BLS. Furthermore, the MMDOM- $f$ and BLS algorithms once again produce relatively close delays, with the former being better in some cases $(200 \leq \bar{\Delta} \leq 1000)$ and the latter in some $(20 \leq \bar{\Delta} \leq 50)$. However, the running time difference of 2-3 orders of magnitude still holds: on average $20 \mathrm{~ms}$ versus 13.8 seconds in the $\bar{\Delta}$ experiments.

In an actual system, the nodal delays (just like the round-trip delay between nodes) are not constant values. Although each node $u$ announces its $\Delta_{u}$ value (short for $\Delta_{u}(1)$, to be precise) as a representative average over time (e.g., exponentially averaged), the momentary nodal delay of $u$ at the time of forwarding our message may be considerably different than the announced average. Neither ours nor previous 


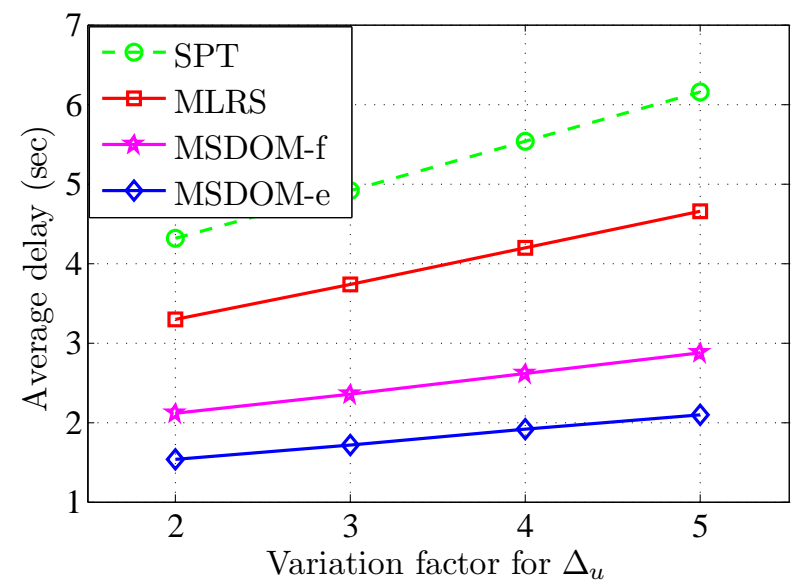

(a) MinSum-delay trees.

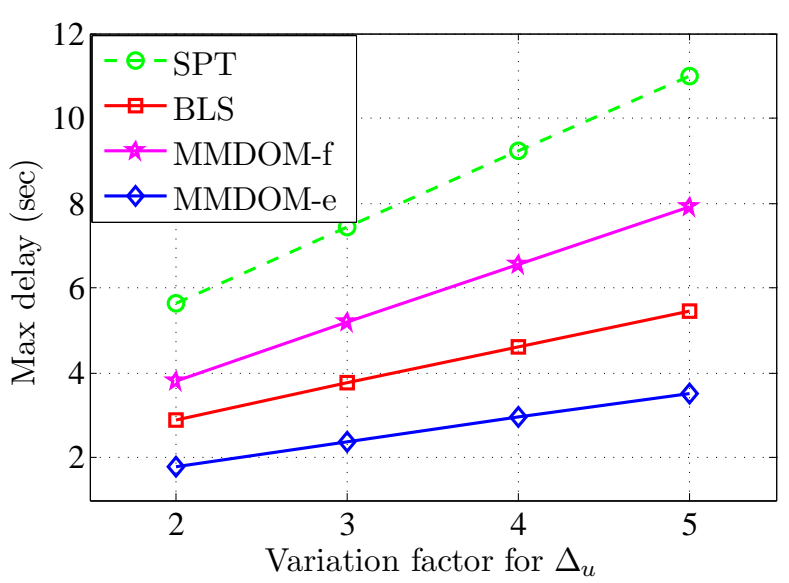

(b) MinMax-delay trees.

Fig. 11. Dynamics of nodal delays $\left(\Delta_{u}\right)$.

algorithms are specifically designed to capture the uncertainty of these delays, though we discuss potential solutions in Section VI. Nevertheless, to have a thorough evaluation study, we analyze the impact of $\Delta_{u}$ dynamics on the efficiency of the different multicast trees. Therefore, right before evaluating a created tree, we set the $\Delta_{u}$ value of each node $u$ to have a random increase or decrease by multiple times: denoting the variation factor of a nodal delay by $v(v=1,2, \ldots)$, we change each $\Delta_{u}$ to a randomly selected value in $\left[\Delta_{u} / v, \Delta_{u} v\right]\left(\Delta_{u}\right.$ is multiplied by $e^{x}$ where $\left.x \sim U(-\ln v, \ln v)\right)$. Figure 11 shows the impact of these dynamics. As expected, the average and maximum delay of all trees rise by the increased fluctuation of nodal delays; we should also note that the average nodal delay increases by $8 \%, 20 \%, 35 \%$, and $50 \%$ for $v=2, \ldots, 5$, respectively. We observe that with varying $\Delta_{u}$ dynamics, the performance of the algorithms relative to each other remains more or less the same, with MSDOM/MMDOM-e algorithms always resulting in the lowest-delay trees.

We also evaluate our algorithms on both datasets $\mathrm{DS}^{2}$ and Meridian, using all the three network models of small world, random, and power law. The results, including tree delays and running times, are presented in Figure 12. The running times for SPT are omitted as they are negligible-few milliseconds to only merge the shortest paths from the routing table. These experiments are conducted on overlays of size $N=1000$ with $R=999, D=100$, and $\bar{\Delta}=100 \mathrm{~ms}$. The experiments for each of the datasets (the first two sets of bars in Figures 12(a) and 12(b)) are conducted on all the three overlay models and the results are averaged. Similarly, the experiments for each overlay model (the last three sets of bars in each figure) are run on both datasets and the average is plotted. We observe in these figures that the different algorithms perform more or less the same as in the previous experiments: MSDOM/MMDOM-e 


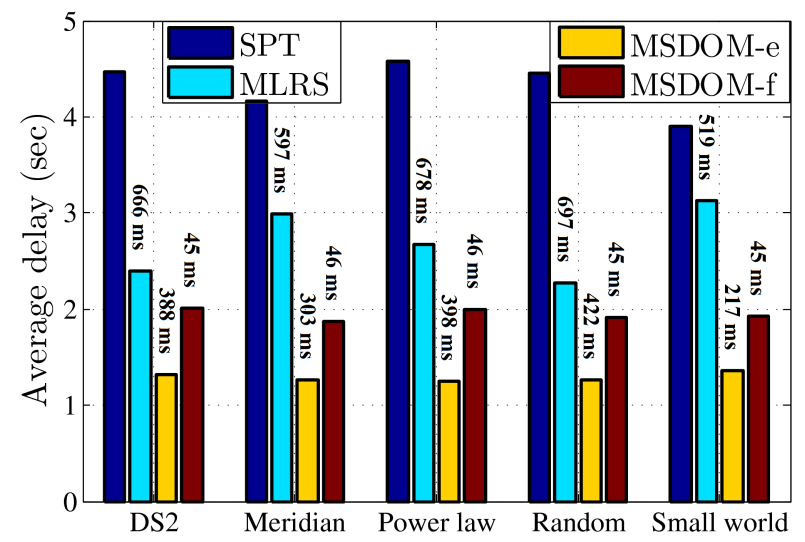

(a) MinSum-delay trees.

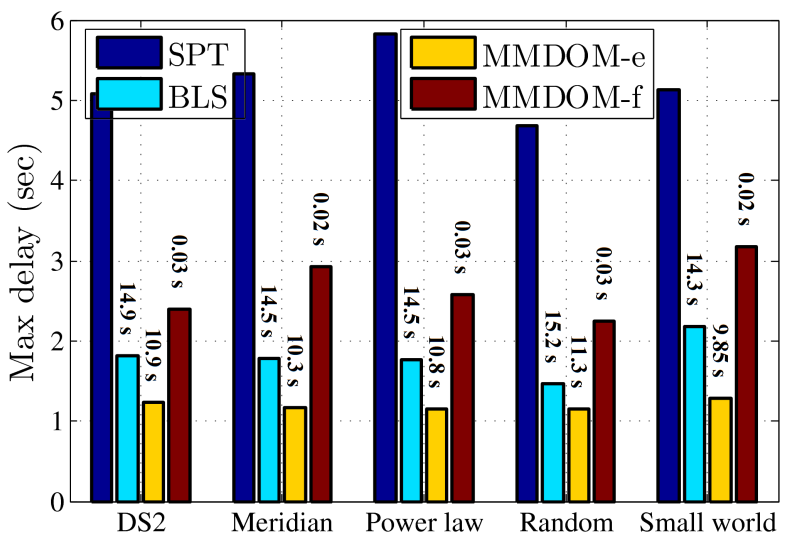

(b) MinMax-delay trees.

Fig. 12. The performance (tree-efficiency as well as running time) of the algorithms on different datasets and overlay models.

algorithms produce the most delay-efficient trees (45-60\% smaller delays than MLRS and 20-40\% than BLS) while also running slightly faster than the previous approaches; note the running times written on top of the bars. MSDOM- $f$ also has better tree efficiency (15-40\%) and running time (over an order of magnitude) than MLRS. MMDOM- $f$ runs in almost zero time for the same inputs on which MMDOM-e and BLS algorithms have taken 10+ seconds, while still creating trees with reasonable delay-efficiencycompare to SPT which is the only relevant alternative.

Summary. We have observed that our algorithms together can support a wide range of overlay sizes for both MinSum and MinMax versions. The MSDOM/MMDOM-e algorithms outperform previous approaches in both tree efficiency and running time, and are the best choice for overlays of 100x nodes. For larger overlays, where previous approaches as well as MSDOM/MMDOM-e are not feasible, MSDOM/MMDOM- $f$ algorithms are suggested which can run fast (100-160 ms for $N=2000$ and 340-490 ms for $N=4000$ in our experiments in Figure 7) while producing trees with reasonable delay efficiencies: $40-55 \%$ smaller delays than SPT trees which are the only applicable alternative. One might also expect that for an overlay of close to 10,000 nodes and up (with reasonable connectivity), path-vector routing may start to become no longer feasible-note the $O(N D L)$ routing information disseminated by each node - and so will be the creation of any multicast tree except the classic SPT. This is because the feasible routing protocol for these scales, distance vector, does not provide any view of the overlay topology but simple distance values. 


\section{CONCLUSiONS}

We have studied the problem of delivering data from a sender to a group of receivers with minimum end-to-end delay in an overlay network. We show that multicast routing algorithms that simply find a shortest-path tree can result in large delays as they only minimize the link-by-link cost, ignoring the important delay incurred at high-degree nodes in the tree. We formulate the problem of minimizing the total and the maximum in a multicast tree, and show that they are NP-hard. We also prove that no polynomial-time approximation algorithm can be found for these problem with a reasonable approximation ratio. We design four algorithms that heuristically create multicast trees with minimum delays: for each of the two minimum-total and minimum-maximum delay cases, we design a delay-efficient algorithm as well as a scale-efficient one (with orders of magnitude faster running time). The collection of these algorithms therefore supports a wide range of overlay scales, from a few hundred to a few thousand nodes. We have conducted a comprehensive evaluation of our algorithms on different real-world dataset and on overlays created from three diverse network models. Our results confirm that our algorithms can achieve significantly lower delays (up to 50+\%) and smaller running times (up to orders of magnitude) than previous minimum-delay multicast algorithms.

We are working on algorithms to only update a previously created multicast tree, rather than re-building it, if the group of receivers has only slightly changed (such as in the example made at the beginning of Section I).

\section{REFERENCES}

[1] P. Davis, "Distributed interactive simulation in the evolution of DoD warfare modeling and simulation," Proceedings of the IEEE, vol. 83, no. 8, 1995.

[2] R. Hofer and M. Loper, "DIS today [distributed interactive simulation]," Proceedings of the IEEE, vol. 83, no. 8, 1995.

[3] D. Schneider, "Trading at the speed of light," IEEE Spectrum, vol. 48, no. 10, pp. 11-12, October 2011.

[4] “Trading shares in milliseconds," MIT Technology Review, February 2010, http://www.technologyreview.com/computing/ 24167.

[5] S. Krause, "A case for mutual notification: a survey of P2P protocols for massively multiplayer online games," in $A C M$ SIGCOMM NetGames'08 Workshop, 2008.

[6] X. Jiang, F. Safaei, and P. Boustead, "Latency and scalability: a survey of issues and techniques for supporting networked games," in IEEE ICN'05, 2005.

[7] D. Ahmed and S. Shirmohammadi, "A dynamic area of interest management and collaboration model for P2P MMOGs," in IEEE/ACM DS-RT'08, 2008.

[8] S. Benford, C. Greenhalgh, T. Rodden, and J. Pycock, "Collaborative virtual environments," Communications of the ACM, vol. 44, no. 7, 2001. 
[9] D. Tran, K. Hua, and T. Do, “A peer-to-peer architecture for media streaming," IEEE Journal on Selected Areas in Communications, vol. 22, no. 1, 2004.

[10] B. Zhang, S. Jamin, and L. Zhang, "Host multicast: a framework for delivering multicast to end users," in IEEE INFOCOM'02, 2002.

[11] M. Hosseini, D. Ahmed, S. Shirmohammadi, and N. Georganas, "A survey of application-layer multicast protocols," IEEE Communications Surveys \& Tutorials, vol. 9, no. 3, 2007.

[12] J. Kurian and K. Sarac, "A survey on the design, applications, and enhancements of application-layer overlay networks," ACM Computing Surveys, vol. 43, no. 1, 2010.

[13] E. Brosh, A. Levin, and Y. Shavitt, "Approximation and heuristic algorithms for minimum-delay application-layer multicast trees," IEEE/ACM Transactions on Networking, vol. 15, no. 2, 2007.

[14] F. Bauer and A. Varma, "Degree-constrained multicasting in point-to-point networks," in IEEE INFOCOM'95, 1995.

[15] P. Jokela, A. Zahemszky, C. Rothenberg, S. Arianfar, and P. Nikander, "LIPSIN: line speed publish/subscribe internetworking," in ACM SIGCOMM'09, 2009.

[16] G. Rodolakis, A. Laouiti, P. Jacquet, and A. Naimi, "Multicast overlay spanning trees in ad hoc networks: Capacity bounds, protocol design and performance evaluation," Computer Communications, vol. 31, no. 7, 2008.

[17] Y. Zhu, C. Dovrolis, and M. Ammar, "Dynamic overlay routing based on available bandwidth estimation: A simulation study," Computer Networks, vol. 50, no. 6, 2006.

[18] H. Zhang, L. Tang, and J. Li, "Impact of overlay routing on end-to-end delay," in ICCCN'06, Arlington, VA, October 2006, pp. 435-440.

[19] D. Andersen, H. Balakrishnan, F. Kaashoek, and R. Morris, "Resilient overlay networks," in ACM SOSP'01, 2001.

[20] Y. Chu, S. Rao, S. Seshan, and H. Zhang, "A case for end system multicast," IEEE Journal on Selected Areas in Communications, vol. 20, no. 8, 2002.

[21] H. Li, L. Mason, and M. Rabbat, "Distributed adaptive diverse routing for voice-over-IP in service overlay networks," IEEE Transactions on Network and Service Management, vol. 6, no. 3, 2009.

[22] Y. Amir, C. Danilov, S. Goose, D. Hedqvist, and A. Terzis, "An overlay architecture for high-quality VoIP streams," IEEE Transactions on Multimedia, vol. 8, no. 6, 2006.

[23] S. Banerjee, B. Bhattacharjee, and C. Kommareddy, "Scalable application layer multicast," in ACM SIGCOMM'02, 2002.

[24] S. Shi, J. Turner, and M. Waldvogel, "Dimensioning server access bandwidth and multicast routing in overlay networks," in ACM NOSSDAV'01, 2001.

[25] S. Banerjee, C. Kommareddy, K. Kar, B. Bhattacharjee, and S. Khuller, "OMNI: An efficient overlay multicast infrastructure for real-time applications," Computer Networks, vol. 50, no. 6, 2006.

[26] W. Zhang, Q. Zheng, H. Li, and F. Tian, "An overlay multicast protocol for live streaming and delay-guaranteed interactive media," Journal of Network and Computer Applications, 2011.

[27] N. Malouch, L. Zhen, D. Rubenstein, and S. Sahu, "A graph theoretic approach to bounding delay in proxy-assisted, end-system multicast," in Proc. of IEEE International Workshop on Quality of Service (IWQoS'02), Miami Beach, FL, May 2002, pp. 106-115.

[28] H. Ito, H. Nagamochi, Y. Sugiyama, and M. Fujita, "File transfer tree problems," in ISAAC'02, 2002.

[29] S. Shi and J. Turner, "Routing in overlay multicast networks," in IEEE INFOCOM'02, 2002.

[30] U. Feige, "A threshold of $\ln n$ for approximating set cover," Journal of the ACM, vol. 45, no. 4, 1998. 
[31] Y. Rekhter, T. Li, and S. Hares, "A border gateway protocol 4 (BGP-4)," Internet Engineering Task Force, RFC 4271, 2006.

[32] C. Wu, B. Li, and S. Zhao, "Exploring large-scale peer-to-peer live streaming topologies," ACM Transactions on Multimedia Computing, Communications, and Applications, vol. 4, no. 3, 2008.

[33] V. Aggarwal, A. Feldmann, and C. Scheideler, "Can ISPs and P2P users cooperate for improved performance?" ACM SIGCOMM Computer Communication Review, vol. 37, no. 3, 2007.

[34] B. Zhang, T. Ng, A. Nandi, R. Riedi, P. Druschel, and G. Wang, "Measurement-based analysis, modeling, and synthesis of the Internet delay space," IEEE/ACM Transactions on Networking, vol. 18, no. 1, pp. 229-242, February 2010.

[35] B. Wong, A. Slivkins, and E. Sirer, "Meridian: a lightweight network location service without virtual coordinates," in Proc. of ACM SIGCOMM'05, Philadelphia, PA, August 2005, pp. 85-96.

[36] S. Lloyd, "Least squares quantization in PCM," IEEE Transactions on Information Theory, vol. 28, no. 2, pp. 129-137, March 1982.

[37] J. Kleinberg, "The small-world phenomenon: an algorithm perspective," in Proc. of ACM Symposium on Theory of Computing (STOC'00), Portland, OR, May 2000, pp. 163-170.

[38] P. Erdos and A. Renyi, “On random graphs,” Publicationes Mathematicae Debrecen, vol. 6, pp. $290-297,1959$.

[39] R. Albert and A. Barabasi, "Statistical mechanics of complex networks," Review of Modern Physics, vol. 74, pp. 47-97, January 2002.

[40] D. Stutzbach, R. Rejaie, and S. Sen, "Characterizing unstructured overlay topologies in modern P2P file-sharing systems," IEEE/ACM Transactions on Networking, vol. 16, no. 2, pp. 267-280, April 2008.

[41] H. Zhang, A. Goel, and R. Govindan, "Using the small-world model to improve Freenet performance," in Proc. of IEEE INFOCOM'02, vol. 3, New York, NY, June 2002, pp. 1228-1237. 